\title{
Correction to: Outcome of MC3 ring annuloplasty for moderate and severe functional tricuspid regurgitation associated with rheumatic mitral valve disease
}

\author{
Shady Eid Al-Elwany, Yasser Shaban Mubarak and Yasser Ali Kamal*i(i)
}

Correction to: Cardiothorac Surg (2019) 27: 5

https://doi.org/10.1186/s43057-019-0007-7

In a recently published article by Al-Elwany et al. [1] in The Cardiothoracic Surgeon, the authors reported that the rate of recurrence of tricuspid regurgitation was $4.3 \%$ after MC3 ring annuloplasty and $23.1 \%$ after DeVega repair. Therefore, we have a correction for a statement in the abstract and section of methods within the text telling that (The rate of TR recurrence $(\geq 2+$ TR) was significantly higher after MC3 ring annuloplasty). The correct statement should be (The rate of TR recurrence $(\geq 2+\mathrm{TR})$ was significantly lower after MC3 ring annuloplasty).

Received: 11 December 2019 Accepted: 16 December 2019

Published online: 30 December 2019

\section{Reference}

1. Al-Elwany SE, Mubarak YS, Kamal YA (2019) Outcome of MC3 ring annuloplasty for moderate and severe functional tricuspid regurgitation associated with rheumatic mitral valve disease. Cardiothorac Surg 27:5. https://doi.org/10.1186/s43057-019-0007-7

\footnotetext{
* Correspondence: Yasser.Ali@mu.edu.eg; yaser_ali_kamal@yahoo.com The original article can be found online at https://doi.org/10.1186/s43057019-0007-7

Department of Cardiothoracic Surgery, Faculty of Medicine, Minia University, El-Minya 61519, Egypt
} 